\title{
Demonstrative study of brain anatomical landmarks by intraoperative ultrasound imaging
}

\author{
Wael Abd Elrahman Ali Elmesallamy
}

\begin{abstract}
Objectives: Intraoperative use of ultrasound in brain surgery needs good understanding of the brain anatomy in ultrasound images. This study aims to compare ultrasound imaging of brain anatomical landmarks during surgery to perioperative computed tomography $(\mathrm{CT})$, and perioperative magnetic resonance imaging (MRI) as demonstration for encouraging usage as low cost, available and hazardless device.

Methods: In total; 350 patients were subjected to brain surgeries under ultrasound guidance using 2.5-8 megahertz (MHZ) transducers, at neurosurgery department Zagazig university hospital from January 2012 to January 2019. Brain anatomical landmarks were compared between ultrasound images, and perioperative images for safe, and confident surgeries.

Results: Various intracranial anatomical landmarks could be well-demonstrated by ultrasound through the open fontanel, or once the skull was opened, and during surgical work in real time fashion, facilitating surgical procedures, and avoiding complications.

Conclusion: Real-time ultrasound is of great help during brain surgeries in delineating brain anatomical landmarks as well as MRI, and CT brain. The growing learning standard of intraoperative ultrasound (IOUS) use makes brain surgery more simple with avoiding brain shift problems, radiation exposure, and high cost of other intraoperative modalities.
\end{abstract}

Keywords: Intraoperative ultrasound, Brain landmarks, Safe surgery, Brain shift

\section{Introduction}

The ultrasound was easy, and excellent in determining brain anatomy, and identifying intracranial abnormalities [6]. Intraoperative ultrasonography (IOUS) is a dynamic imaging that provides real-time information during craniotomy [9]. Historically; the neurosurgeon to calculate the tumor location used the crude, and ambiguous visual, and tactile methods (gyri widening, discoloration, and tissue consistency), and may do blind exploration to localize subcortical lesions with risk of error [13].

Neuro-navigator systems based on preoperative data are not accurate due to brain shift [4]. The imaging artifacts, and ionizing radiation as well as limited tumor definition limited the use of intraoperative CT.

\section{Correspondence: waelmesallamy@gmail.com}

Neurosurgery Department, Faculty of Medicine, Zagazig University, Zagazig, Egypt

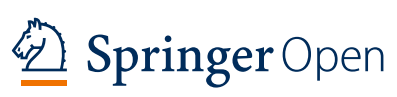

Intraoperative MRI prolongs the time of surgery beside high costs, and special surgical equipment [3]. The best imaging modalities for brain shift compensation are IOUS, and intraoperative MRI [2].

As unfamiliar image planes, and lack of experience; the ultrasound images can be difficult to interpret [1]. IOUS is easy to use, and provides the real-time visualization of brain anatomical structures [12].

I attempted to assess the ability of intraoperative ultrasound in brain surgery to detect brain landmarks, and compare with perioperative imaging for safe surgical procedures and collect data as clinical demonstrative study.

\section{Patients and methods}

This is a qualitative study to demonstrate important brain anatomical landmarks faced during intraoperative 
use of ultrasonography in brain surgeries. In total; 350 patients were subjected to brain surgeries either for mass lesions resection (supratentorial or infratentorial) or ventriculoperitonial shunt insertion under ultrasound guidance; during the period from January 2012 to January 2019 at neurosurgery department, Zagazig university hospitals after approval from the local ethical committee and Zagazig university institutional review board ( $\mathrm{Zu}-$ IRB). Informed consent according to the criteria set by the local research ethics committee in our center had to be obtained in writing before surgery. If consent could not be obtained because the patient was in coma, or dysphasic, or a child; consent was obtained from relatives for the procedures. All patients were operated upon under general anesthesia in proper operative position.

Conventional ultrasound IBE-2500D, digital scanner with electronic endocavity transducer (5, 6.5 and $8 \mathrm{MHZ}$ ), and electronic convex array transducer $(2.5,3.5$ and 5 MHZ) was used. The probe was placed on the opened fontanel during ventriculoperitoneal (V-P) shunt insertion. During mass lesion surgery the probe placed on the dura, then on the brain, and in the resection cavity during resection progression of the mass lesions. The probe was covered by a sterile glove, and acoustic gel was putted inside the glove. Low frequencies were used for deep details while high frequencies for superficial details.

The cavity during mass resection was filled by saline during use of the ultrasound probe. The real time mode was used, and then B-mode for doing the measurements.

Tissues that are more echogenic than brain tissues appear more white on the screen, and the less echogenic appear more dark. For delineation of the best image of the brain, and the mass lesion; the following factors must be applied:

- Position of the patient head allows the lesion, and the resection cavity are upward to permit saline stay in the resection cavity, and prevent air artifact. In posterior fossa surgery in sitting position elongated glove gel pad was used to fill the surgical cavity.

- Selecting the proper ultrasound probe size, and frequencies.

- Gel connection without air bubbles inside the glove.

- Adjusting the scanner brightness, depth, gain compensation, focus, and frequency for best images.

- Cottonoids, and spatula should be removed, and blood washing by saline during imaging.

\section{Results}

This study included 350 patients; 30 patients operated for ventriculopretonial shunt insertion of ages less than two years, with open anterior fontanel and hydrocephalus, and 320 patients suffer brain mass lesions either supratentorial or infratentorial. The ages of the patients ranged from 1 months to 71 years old with mean of 45.2, and 231 of them were males (66\%). The mass lesion natures were; brain tumors in 236 patients, abscesses in 30 patients, and hematomas in 54 patients; Table 1 which showed also the operative complications.

The ultrasound gave us a real time image without error of brain shift of the neuronavigators which depend on preoperative images. The echogenicity of the brain landmarks, its type, shape, and relation to the mass lesion were evaluated for safe surgery according to the location of the mass lesion Table 2. It is of great importance especially for lesions entangling the ventricles or an eloquent brain area for safe trajectory angulation. The nearby brain landmarks differ according to the mass lesion location, and must be reevaluated during surgical progression Figs. 1, 2, 3, 4, 5 and 6.

\section{Discussion}

Availability of ultrasound was before CT, and MRI, and its performance does not require any major investment. Routinely available ultrsaounds are good, and enough; only choose the size of the probe, and frequency for work adaptation. This study tried to describe the brain landmarks faced during brain surgery under ultrasound B-mode which of great help in surgical orientation for safe surgery.

Routine scanners available in hospitals are sufficient in brain surgeries [11]. Brain tissue deformation, and brain

Table 1 Patients and brain lesions data

\begin{tabular}{lll}
\hline Parameters & No & $\%$ \\
\hline Age & 112 & 32 \\
Child $\leq 18$ years old & 238 & 68 \\
Adults > 18 years old & & \\
Sex & 231 & 66 \\
Male & 119 & 34 \\
Female & & \\
Location & 237 & 74 \\
Supratentorial & 83 & 26 \\
Infratentorial & & \\
Pathology & 30 & 8.6 \\
Hydrocephalus & 236 & 67.4 \\
Tumors & 54 & 15.4 \\
Hematoma & 30 & 8.6 \\
Abscess & & \\
Complications & 2 & 0.6 \\
Operative death & 9 & 2.6 \\
Transient complications & 7 & \\
Persistant complications & 2 & \\
Infections & & \\
\hline
\end{tabular}


Table 2 Brain landmarks by intraoperative ultrasound

\begin{tabular}{lll}
\hline Brain landmark & $\begin{array}{l}\text { Echogenicity according to Gray-white scale in relation to brain } \\
\text { tissues }\end{array}$ & Description \\
\hline Ventricles & Homogeneous hypo-echogenic & Centrally located \\
Choroid plexus & Homogeneous hyper-echogenic & Ribbon shape inside the ventricles \\
Septum pellucidum & Homogeneous hyper-echogenic & Thick continuous line \\
Sulci & Heterogeneous hyper-echogenic & Small irregular dipping lines \\
Cistern & Heterogeneous hyper-echogenic & Lined and marginated cavity \\
Inter-hemispheric & Heterogeneous hyper-echogenic & Large continuous thick line with side branching \\
fissure & & \\
Falx cerebri & Homogeneous hyper-echogenic & Thick continuous line may be shifted by mass effect \\
Tentorium cerebelli & Homogenous hyper-echogenic & Thick continuous line \\
Brain tissues & Homogeneous iso-echogenic & Difficult to delineate white from gray matters \\
Brain stem & Heterogeneous hyper-echogenic or hypo-echogenic & Rectangular in shape \\
Skull & Homogeneous hyper-echogenic & Thick continuous line \\
Edematous brain & Heterogeneous hyper-echogenic or hypo-echogenic & According to severity, more hypo-echogenic with tense \\
& & edema
\end{tabular}

shift during surgery are the main limitation of neuronavigator, which depends on preoperative imaging. Ultrasound is a real time modality, but most neurosurgeons trained on CT, and MRI, lack ultrasound training and have difficulties in recognizing anatomic structures in comparison to preoperative imaging [16]. Ultrasound images were comparable to or even better than the corresponding MRI scans regarding tumor, and landmark visualization [18]. There are no real disadvantages with the use of intraoperative ultrasound. No risk of mechanical brain injury or infection if used properly. Ultrasound is easily available, convenient, fast, simple to use, and cheap [13].

The ultrasound images echogenicity is related to mass density, and ultrasound propagation in diverse tissues [8]. Hypo-echoic lesions have an elevated water component, whereas hyperechoic lesions are characterized by a rich capillary network or a significant stromal component [14].

Because intraoperative ultrasound gives imaging of normal structures adjacent to the lesion, the relationship to these normal structures can be appreciated before, during, and after resection [5].

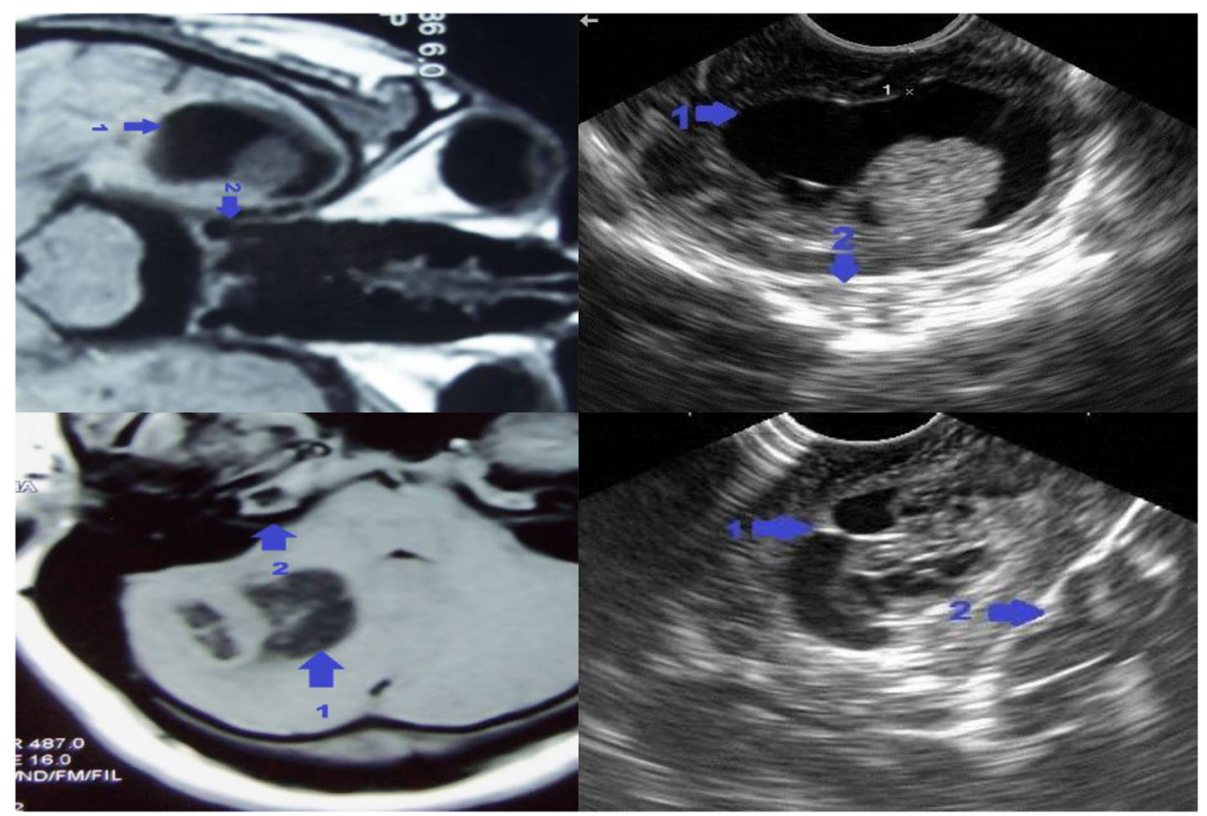

Fig. 1 Bone; arrow1 brain mass lesion, arrow2 bone (homogeneous hyper-echogenic) 


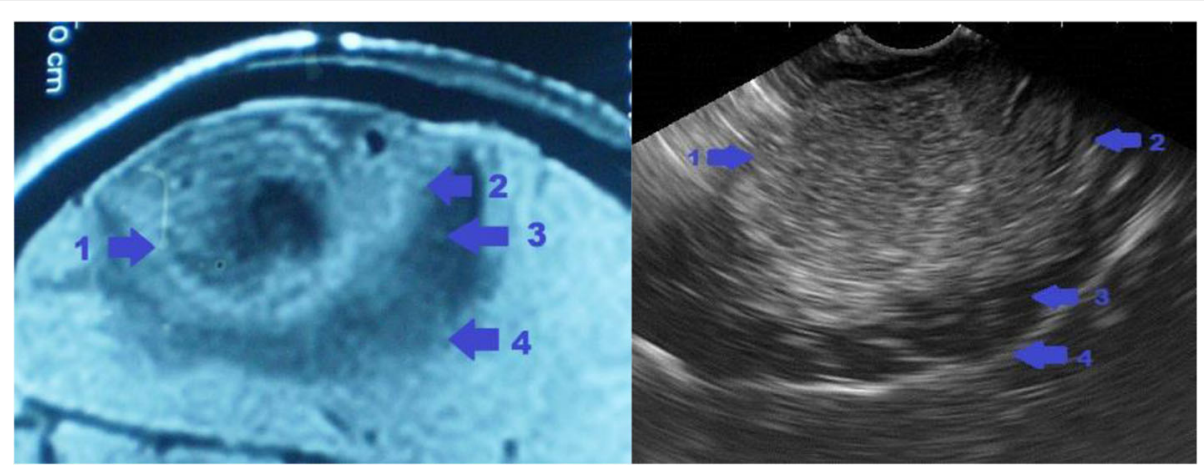

Fig. 2 Brain edema; arrow1 brain mass lesion, arrow2 heterogeneous hyper-echogenic brain edema, arrow3 hypo-echogenic brain edema, and arrow4 interface between brain and edema

Ultrasound, MRI, and CT images do not emphasize the same physical properties of the tissues so; have markedly different appearance. In this study; important anatomical landmarks detected well by intraoperative ultrasound. Echogenicity relative to brain tissues and homogeneity beside shape and location gave visual orientation to brain anatomical landmarks as well as brain pathology. Brain stem, ventricular system, sulci, cisterns, and other landmarks showed in illustrated Figs. 1, 2, 3, 4, 5 and 6, with comparison to other imaging modalities.

Several studies described ultrasound anatomical landmarks, but without demonstration and concentration on this subject. Park et al [15]; found the ventricles appear homogeneous hypo-echogenic less than the homogeneous brain tissues. The choroid plexus appears homogeneous hyper-echogenic mass, while the falx cerebri, the tentorium cerebelli, and the bone appear homogenous hyper-echogenic lines. The interhemispheric fissure, and brain sulci appears heterogeneous hyperechogenic lines. Wang et al [20]; mentioned that the meninges intruding into brain tissue were seen as hyperechoic. Panagiota, and Pallikarakis [14]; described the surface of normal brain sulci to be moderately echogenic, while the deeper tissues are quite hypo-echoic,

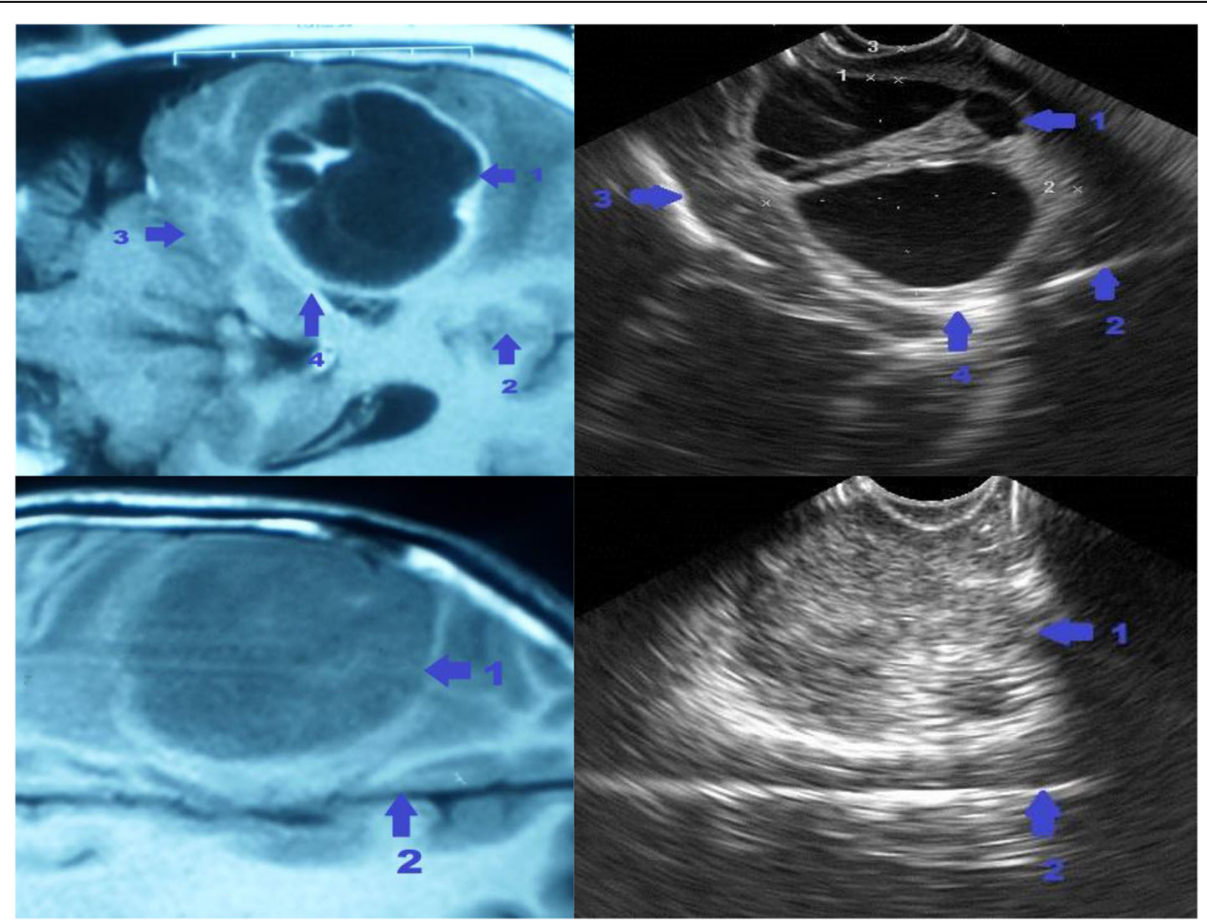

Fig. 3 Brain dura and choroid plexus; arrow1 brain mass lesion, arrow2 homogenous hyper-echogenic falx cerebrai, arrow3 homogenous hyperechogenic tentorium cerebelli, and arrow4 homogenous hyper-echogenic choroid plexus 


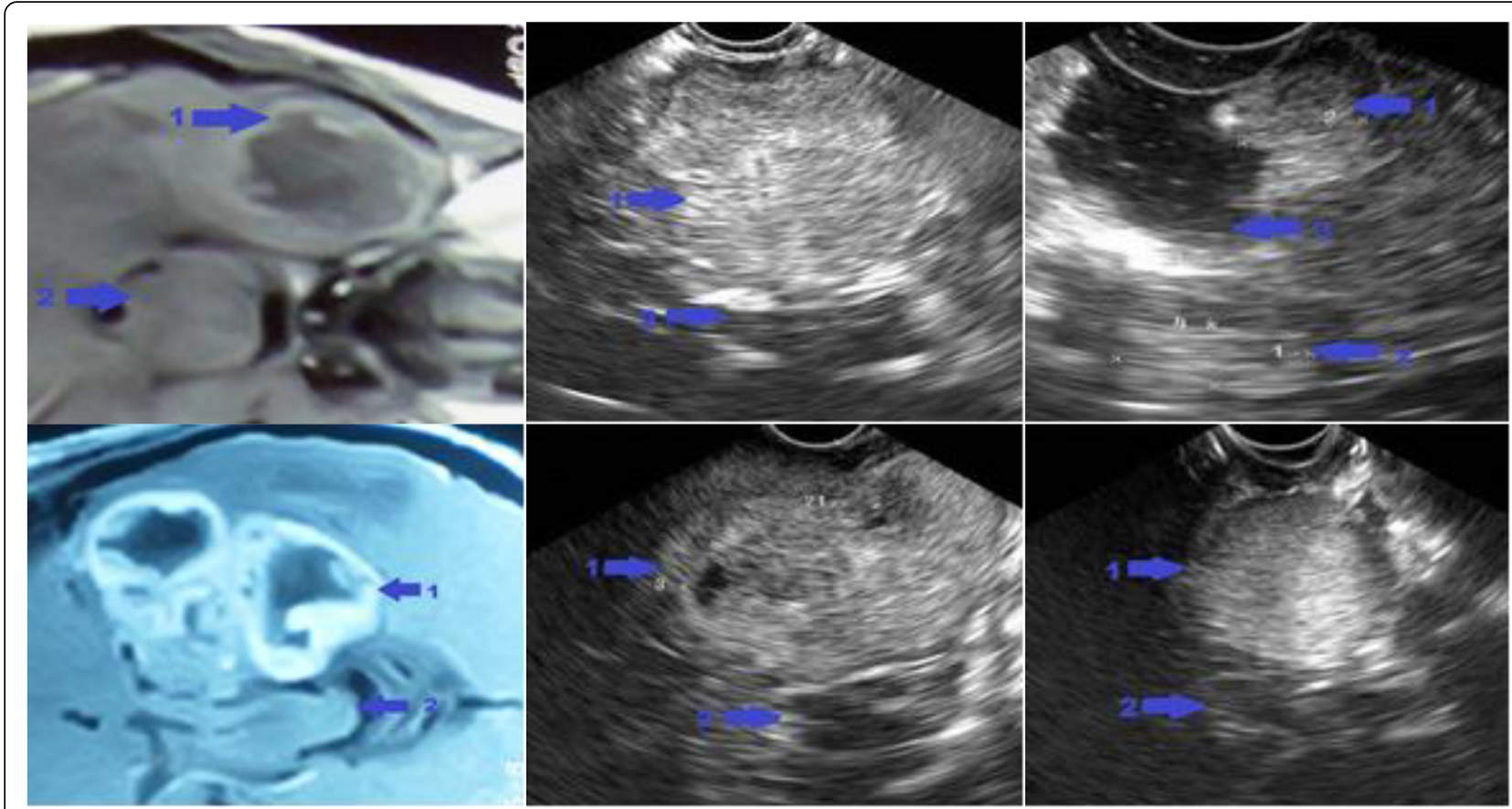

Fig. 4 Brain stem; arrow1 brain mass lesion, arrow2 brain stem (heterogeneous hypo-echogenic or hyper-echogenic), and arrow3 resection cavity

and homogenous. The ventricles are a good centrally located landmark due to their homogeneous hypoechogenicity. On contrary, the sulci, the falx, and choroid plexus are heterogeneously hyper-echogenic. Cheon [7]; described the ventricles as anechoic, while the falx, and arachnoid membrane as echogenic structures. Chen et al [6]; described the brain stem as homogenous hypoechogenic due to compact nerve bundles, and nuclei. Also, Chandler, and Rubin [5]; mentioned that the brain stem in ultrasound image is typically hypo-echoic.
Edematous brain, surrounding brain mass lesion appeared hyper-echogenic, but when tense it appeared hypo-echogenic. Many authors described brain edema as more echogenic than the normal brain as $[6,10,17,19]$. Panagiota, and Pallikarakis [14]; described the transition between the lesion, and healthy brain by sharp or mediated by a hypo-echoic or hyper-echoic halo. Hypoechoic halo can be the expansion of perifocal compressive edema, perilesional vessels, or a tumor component with active growth.

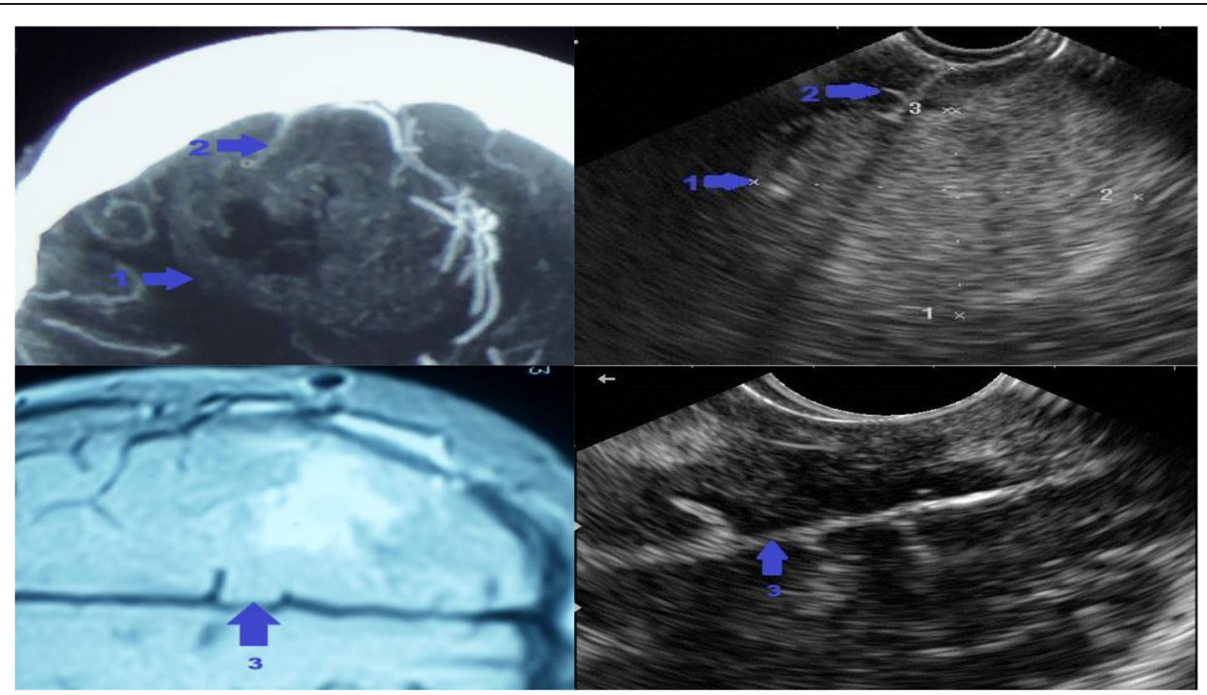

Fig. 5 Brain subarachnoid spaces; arrow1 brain mass lesion, arrow2 heterogeneous hyper-echogenic brain sulcus, arrow3 heterogeneous hyperechogenic inter-hemispheric fissure and arrow4 heterogeneous hyper-echogenic cistern 


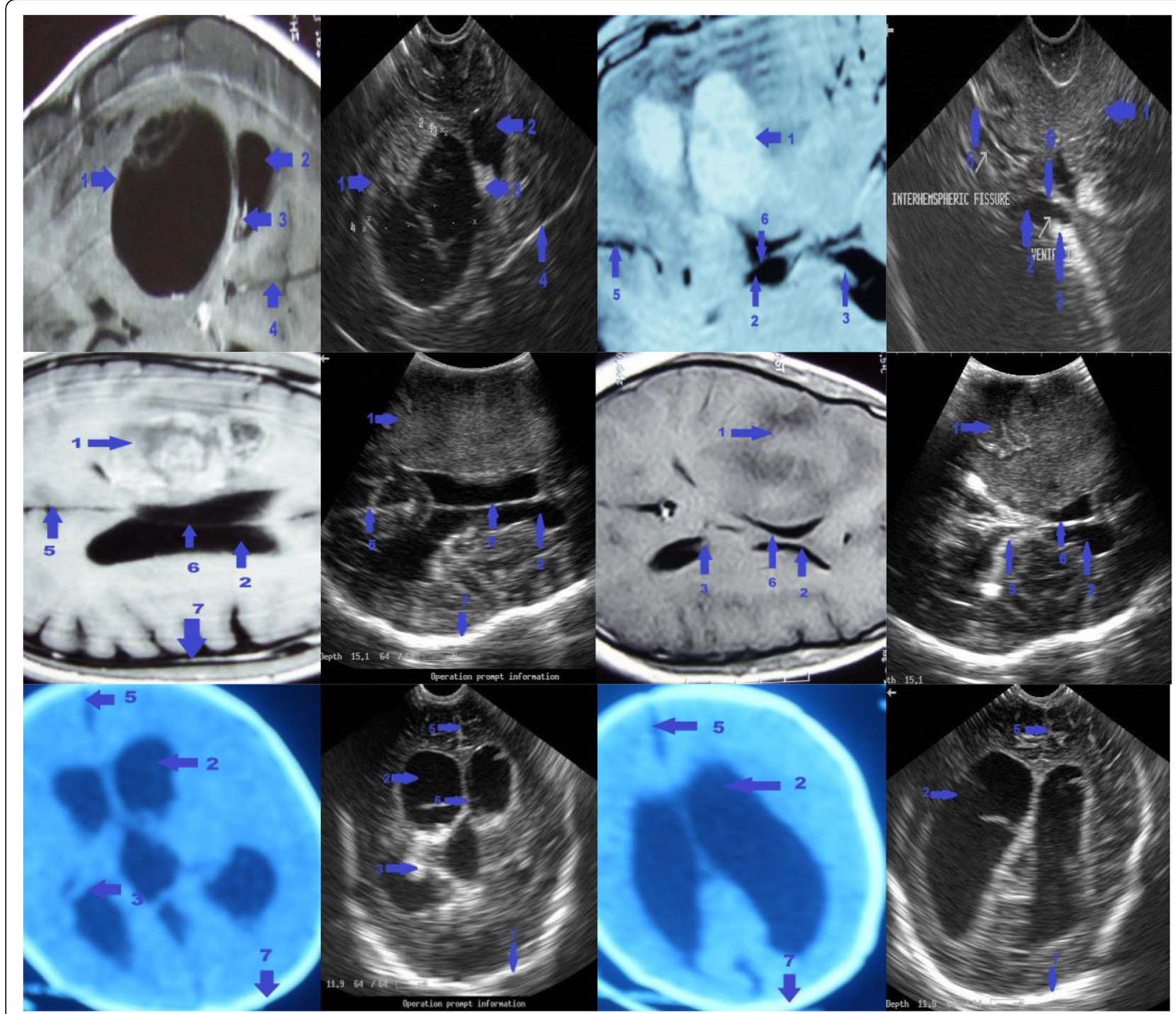

Fig. 6 Ventricular system; arrow1 brain mass lesion, arrow2 homogenous hypo-echogenic cerebrospinal fluid, arrow3 homogenous hyperechogenic choroid plexus, arrow4 homogenous hyper-echogenic falx, arrow5 heterogeneous hyper-echogenic inter-hemispheric fissure, arrow6 homogenous hyper-echogenic septum pellucidum, and arrow 7 homogenous hyper-echogenic bone

\section{Conclusion}

Intraoperative ultrasound is a reliable method for visualization of important brain landmarks during brain mass lesion surgeries, and ventriculoperitoneal shunt insertion, and this helps safe surgery, and confident intraoperative decisions for saving the patient, and evaluation of the surgical process. It is easy to learn how to interpret the ultrasound image as well as our familiar CT, and MRI images for encouraging the use of this simple, cheap, and valuable machine.

\section{Study limitations}

The used IOUS in this study not adapt 3diminsion capability or vascular modes except $\mathrm{M}$ mode.

\section{Abbreviations}

CT: Computed Tomography; IOUS: Intra-Operative Ultrasound; MHZ: Megahertz; MRI: Magnetic Resonance Imaging; V-P shunt: Ventriculoperitoneal shunt

\section{Acknowledgements}

It is lucky to work with the neurosurgery team at Zagazig university hospital for great help and support.

\section{Author's contribution}

The author contributed to the study conception, design, most surgical works, data collection and drafting the manuscript. The author read and approved the final manuscript.

\section{Funding}

All patients operated at Zagazig university hospitals neurosurgery department for free. 


\section{Availability of data and materials}

All data that support the findings of this study are available from the neurosurgery department Zagazig university hospital. Data are however available from the author when requested with permission.

\section{Ethics approval and consent to participate}

A research committee approval has been granted for this study by the medical ethics committee, Faculty of Medicine, Zagazig university at 5/1/ 2012. Informed consent according to the criteria set by the local research ethics committee in our center obtained in writing before surgery. If consent could not be obtained because the patient was in coma or young age $(<18$ years), consent was obtained from relatives.

\section{Consent for publication}

Informed consents were obtained for all patients and no personal data included in this study.

\section{Competing interests}

The author declares that he has no competing interests.

Received: 22 May 2019 Accepted: 16 September 2019

Published online: 09 October 2019

\section{References}

1. Andrews BT, Mampalam TJ, Omsberg E, Pitts LH. Intraoperative ultrasound imaging of the entire brain through unilateral exploratory burr holes after severe head injury: technical notes. Surg Neurol. 1990;33:291-4.

2. Bayer S, Maier A, Ostermeier M, Fahrig R. Intraoperative imaging modalities and compensation for brain shift in tumor resection surgery. Int J Biomed Imaging. 2017(3):1-18.

3. Bernays RL. Intraoperative imaging in neurosurgery: MRI, $C T$ and ultrasound Introduction. Acta Neurochir Suppl. 2003;85:1-3.

4. Bonsanto MM, Staubert A, Wirtz CR, Tronnier V, Kunze S. Initial experience with an ultrasound-integrated single-rack neuronavigation system. Acta Neurochir. 2001;143(11):1127-32.

5. Chandler WF, Rubin IM. The application of ultrasound during brain surgery. World J Surg. 1987:11:558-69.

6. Chen SY, Chiou TL, Chiu WT, Su CF, Lin SZ, Wang SG, Yen PS. Application of intraoperatiev ultrasound for brain surgery. Tzu Chi Med J. 2004;16(2):85-92.

7. Cheon JE. Intraoperative neurosonography revisited: effective neuronavigation in pediatric neurosurgery. Ultrasonography. 2015;34(2):79-87.

8. Dohrmann GJ, Rubin JM. History of intraoperative ultrasound in neurosurgery. Neurosurg Clin N Am. 2001;12:155-66.

9. Kane RA, Kruskal JB. Intraoperative ultrasonography of the brain and spine Ultrasound Q. 2007;23:23-39.

10. Knake JE, Silver TM, Chandler WF. Intraoperative cranial sonography for neurosurgical procedures. Semin Ultrasound. 1982(2):200-8.

11. Mari AR, Shah I, Imran M, Ashraf J. Role of intraoperative ultrasound in achieving complete resection of intra-axial solid brain tumors. J Park Med Assoc. 2014:64:1343-7.

12. Mbuyamba IE, Linder D, Cervantes AJG, Arlt F, Rostro H, Cruz I, Chalopin C. Fusion of intraoperative 3 D B-mode and contrast-enhanced ultrasound data for automated identification of residual brain tumors. Appl Sci. 2017; 7(415):1-17

13. Moiyadi A, Shetty P. Objective assessment of utility of intraoperative ultrasound in resection of central nervous system tumors: a cost-effective tool for intraoperative navigation in neurosurgery. J Neurosci Rural Practice. 2011;2:4-11

14. Panagiota K, Pallikarakis N. Simulation of ultrasound brain cancer imaging In: Master's Thesis University of Patras, Faculty of Medicine; 2011. p. 1-108.

15. Park J, Woo H, Kim GCH. Diagnostic usefulness of intraoperative ultrasound for unexpected severe brain swelling in ultra-early surgery for rupture intracranial aneurysm. Acta Neurochir. 2012;154:1869-75.

16. Prada F, Delbene M, Mattei L, Dimeco F. Preoperative magnetic resonance and intraoperative ultrasound fusion imaging for real time neuronavigation in brain tumor surgery. Eur J Ultrasound. 2015;36(2):174-86.

17. Rogers JV, Shuman WP, Hirsch JH, Lang S, Howe J, Burchiel K. Intraoperative neurosonography: application and technique. AJNR. 1984;5:755-60.

18. Unsgaard G, Ommendal S, Muller T, Gronningsaeter A, Hernes TAN. Neuronavigation by intraoperative three-dimensional ultrasound: initial experience during brain tumor resection. Neurosurgery. 2002;50(4):804-12.
19. Wang J, Duan YY, Liu X, Wang Y, Gao GD, Qin HZ, Wang L. Application of intraoperative ultrasonography for guiding microneurosurgical resectin of small subcortical lesions. Korean J Radiol. 2011;12(5):541-6.

20. Wang J, Liu X, Hou WH, Dong G, Wei Z, Zhou H, Duan YY. The relationship between intraoperative ultrasonography and pathological grade in cerebral glioma. J Int Med Res. 2008;36:1426-34.

\section{Publisher's Note}

Springer Nature remains neutral with regard to jurisdictional claims in published maps and institutional affiliations.

\section{Submit your manuscript to a SpringerOpen ${ }^{\circ}$ journal and benefit from:}

- Convenient online submission

- Rigorous peer review

- Open access: articles freely available online

- High visibility within the field

- Retaining the copyright to your article

Submit your next manuscript at $\boldsymbol{\nabla}$ springeropen.com 